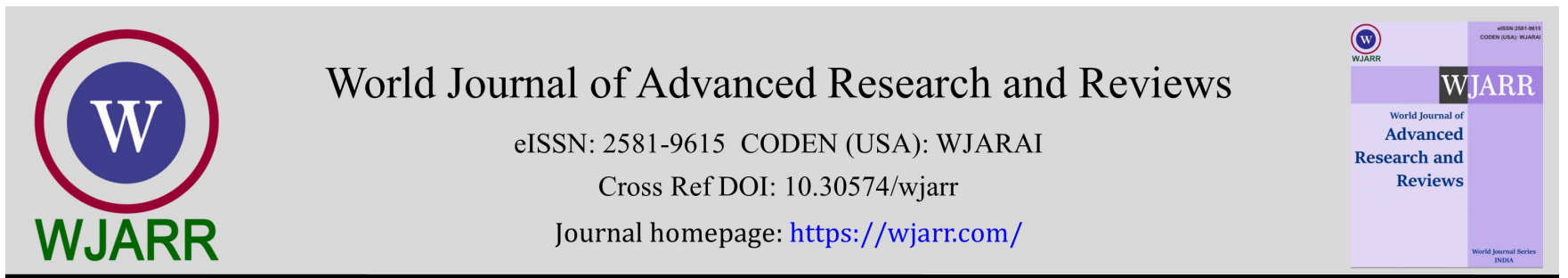

(RESEARCH ARTiClE)

Check for updates

\title{
Study of the effect of direct acting antivirals (DAAs) on fibrosis in chronic hepatitis C patients using transient elastography
}

\author{
Alaa Aboud Mohammed 1, *, Dina Attia 1, Basel Abdelmonem Ebeid ${ }^{1}$ and Mariam Boulous Ibrahim ${ }^{2}$ \\ ${ }^{1}$ Department of Gastroenterology, Hepatology and Endemic Medicine, Faculty of medicine Beni-Suef University, Egypt. \\ ${ }^{2}$ Faculty of Medicine, Minia University, Egypt.
}

World Journal of Advanced Research and Reviews, 2021, 10(02), 135-145

Publication history: Received on 05April 2021; revised on 08May 2021; accepted on 11 May 2021

Article DOI: https://doi.org/10.30574/wjarr.2021.10.2.0213

\begin{abstract}
Back ground: As DAA agents are leading to enormous cure rates, so identifying and monitoring patients who remain at a high complication risk after achieving SVR continue to be a critical issue. Numerous validated methods for noninvasive measurement of liver fibrosis can be used in the management of HCV infection.

Methods study: A total of 50 Egyptian chronic HCV patients eligible for treatment with DAAs were enrolled in this prospective study. All subjects selected from Virology Unit of Tropical Medicine Department, Faculty of Medicine, BeniSuef University received DAAs (SOV DCV) the endpoint was a sustained virologic response at 12 (SVR12) weeks posttreatment. All participants were evaluated non-invasively by Liver Stiffness Measurement (LSM) by Fibro Scan before DAAs treatment, and 6 months post-treatment.
\end{abstract}

Results: SVR achieved by DAAs therapy was $96 \%$ and was associated with significant improvement $(p<0.05)$ of noninvasive fibrosis markers (FIB-4, APRI score, and LSM by Fibro Scan) from baseline compared to 6 months posttreatment. Using fibro scan regression of fibrosis in 30/45 patients (66\%). Liver inflammation and synthetic functions also improved on follow up $12 \mathrm{w}$ and $6 \mathrm{~m}$ post treatment.

Conclusion: Regression of Liver inflammation and fibrosis in chronic hepatitis C patients post treatment with SOF DCV

Keywords: DAAs; Fibrosis; Fibro scan

\section{Introduction}

Hepatitis $\mathrm{C}$ virus (HCV) is a worldwide etiology of chronic hepatic insult particularly in Egypt where the main genotype is genotype 4 which is responsible for $90 \%$ of infections and the remaining is due to genotype-1 (1)

In recent years, the treatment of HCV has been evolved with the development of direct acting antiviral (DAAs) therapies, and they have entered the clinical practice in 2014/2015. They showed a promising future for HCV treatment with higher SVR rates, shortened and simplified regimens, and minimal treatment-related side effects in HCV patients (2).

As DAA agents are leading to enormous cure rates, so identifying and monitoring patients who remain at a high complication risk after achieving SVR continue to be a critical issue. Numerous validated methods for noninvasive measurement of liver fibrosis can be used in the management of HCV infection. Measurement of hepatic fibrosis

${ }^{*}$ Corresponding author: Alaa Aboud Mohammed

Assistant professor of tropical medicine, faculty of medicine Beni suef university EGYPT.

Copyright (C) 2021 Author(s) retain the copyright of this article. This article is published under the terms of the Creative Commons Attribution Liscense 4.0. 
noninvasively enables the identification of at-high risk patient without the need for liver biopsy. The application of the noninvasive techniques produced a simplified approach in the management of patients with chronic HCV infection. (3).

Transient elastography (TE) is the most widely used and validated technique that measures liver stiffness. It was the first ultrasound-based elastography technique used in clinical practice, and is currently recommended by international guidelines for the evaluation of patients with chronic liver diseases, and it was also approved by the Egyptian National Committee for Control of Viral Hepatitis (NCCVH) to replace liver biopsy in evaluating liver stiffness in chronic HCV patients (4)

\section{Patients and methods}

\subsection{Type of the study}

This study is a prospective study.

\subsection{Site of the study}

Virology Unit of Tropical Medicine Department, Faculty ofMedicine, Beni-Suef University.

\subsection{Date and Period of the study}

The study started from January 2019.

\subsection{Study population}

The study will be done on chronic hepatitis c patients on directacting antivirals (DAAs) , attending the Virology Unit ofTropical Medicine Department, Faculty of Medicine, Beni-SuefUniversity.

\subsection{Sample size}

The study will be conducted on 50 chronic hepatitis c patientsstarting treatment on direct acting antivirals and will beselected randomly according to the inclusion and exclusioncriteria.

\subsubsection{Inclusion Criteria:}

- Chronic hepatitis c virus patients starting on direct acting antivirals (DAAs).

- Age $>18$ years.

- Compensated cirrhotic patients (Child-Pugh A)

- Hepatocellular carcinoma patients after six months oftreatment.

- Extra hepatic malignancy after two years of treatment.

- Bilirubin $<3$

- Albumin $>2.8$

- $\quad$ Platelets $>50000$

- $\quad$ INR $<1.7$

\subsubsection{Exclusion Criteria:}

- $\quad$ Child B and C cirrhotic patients.

- Age < 18 years.

- Pregnancy or inability to use effective contraception.

- Hepatocellular carcinoma patients before six months oftreatment.

- Extra hepatic malignancy before two years of treatment.

- Bilirubin $>3$

- Albumin $<2.8$

- Platelets $<50000$

- $\quad$ INR $>1.7$

- Dilated biliary radicles.

- Cardiac decompensation.

- $\quad$ BMI > 35

- Ascites. 
- Patients not fasting.

- Patients refused to be included in the study.

\subsection{Methods:}

All selected patients will be subjected to:

- History taking

- Abdominal examination

- 3-Investigations :

a- Laboratory investigations including ALT (SGPT), AST (SGOT), ALP, Alumin, Bilirubin, prothrombin time, Alfa fetoprotein.

b- Imaging including abdominal ultrasonography for:

- $\quad$ Liver : texture, size, hepatic vein, portal vein and collaterals

- Spleen: size, splenic vein diameter.

- Ascites.

c- Transient Elastography will be performed by a single competent operator who blindly dealing with the patients regardless their clinical and laboratory data. It will be made to patients twice, before starting treatment and after six months of treatment to know the effect of treatment on fibrosis in these patients. Patients are fasting for at least $6 \mathrm{~h}$ before examination, patient lie in the dorsal decubitus with the Rt arm in maximal abduction, Measurement are taken in the Rt lobe of liver by placing the tip of the transducer perpendicularly in between the 9 th to 11 th intercostalspaces.

The device that will be used in the study is fibro scan 502 .

d- Upper endoscopy if indicated.

Investigations:

- Baseline:

- $\quad$ ALT (SGPT), AST (SGOT), Albumin, Bilirubin.

- Complete blood count.

- Abdominal ultrasonography.

- Transient Elastography.

- If patient age > 65 years, ECG and Echocardiography will bedone.

Follow up: -

ALT (SGPT), AST (SGOT), Albumin, Bilirubin, and CBC will be repeated after 4 weeks, 8 weeks,and 12 weeks from the beginning oftreatment and 4 weeks, 12 weeks after the end of treatment thentransient elastography will be repeated.

\subsection{Ethical Consideration and Review:}

Study protocol will be approved from The National Committeefor control of Viral Hepatitis and Local Ethical Committeeof Faculty of Medicine, Beni-Suef University. Data will beconfidential and anonymous. Informed written consent will betaken from all studied patients.

\subsection{Statistical Analysis}

The collected data will be coded then entered and analyzedusing statistical package for the Social Sciences Program(SPSS) version 21. Both descriptive and inferential dataanalysis will be employed. Comparison of quantitative data willbe assessed by student test. Comparison of qualitative data willbe assessed by Chi-square test. In all tests, $\mathrm{P}$ was consideredsignificant if $<0.05$. 


\section{Results}

Table 1shows that the mean age of the study group was $54.6 \pm 4.1$ years. Male sex was predominating among the study population $(71.1 \%) .5$ patients were missed during follow up.

Table 1 Demographic data of the study group

\begin{tabular}{|l|l|l|l|l|}
\hline & Minimum & Maximum & Mean & Std. Deviation \\
\hline Age(years) & 46.00 & 60.00 & 54.6444 & 4.11293 \\
\hline Sex & Number & Percent & \\
\hline Males & 32 & 71.1 & \\
\hline Females & 13 & 28.9 & \\
\hline
\end{tabular}

Table 2 Comparison between laboratory data before and after treatment.

\begin{tabular}{|l|l|l|l|l|}
\hline $\begin{array}{l}\text { Laborat } \\
\text { ory data }\end{array}$ & Beforetreatment & Aftertreatment & $\begin{array}{l}\text { After12 weeksof } \\
\text { treatment }\end{array}$ & P \\
\hline $\mathrm{Hb}$ & $13.182 \pm 0.628$ & $13.282 \pm 0.632$ & $13.062 \pm 0.651$ & 0.44 \\
\hline PLT & $207.222 \pm 47.734$ & $225.600 \pm 52.070$ & $235.111 \pm 55.501$ & 0.038 \\
\hline TLC & $5.035 \pm 0.278$ & $5.068 \pm 0.435$ & $5.080 \pm 0.455$ & 0,56 \\
\hline ALT & $68.266 \pm 23.006$ & $39.022 \pm 15.024$ & $38.222 \pm 22.702$ & $<0.001$ \\
\hline AST & $59.844 \pm 17.266$ & $34.222 \pm 13.777$ & $32.977 \pm 17.479$ & $<0.001$ \\
\hline Albumin & $4.255 \pm 0.584$ & $4.704 \pm 0.364$ & $4.720 \pm 0.452$ & $<0.001$ \\
\hline Bilirubin & $1.077 \pm 0.318$ & $0.884 \pm 0.152$ & $0.902 \pm 0.216$ & $<0.001$ \\
\hline $\begin{array}{l}\text { Creatini } \\
\text { ne }\end{array}$ & $1.052 \pm 0.125$ & $1.028 \pm 0.131$ & $1.012 \pm 0.139$ & 0.16 \\
\hline INR & $1.056 \pm 0.141$ & $0.981 \pm 0.110$ & $0.961 \pm 0.131$ & 0.003 \\
\hline AFP & $3.362 \pm 1.458$ & $2.811 \pm 1.306$ & $2.556 \pm 1.331$ & 0.019 \\
\hline
\end{tabular}

Table 2 show significant improvement of liver enzymes, liver synthetic functions (Albumin, bilirubin, INR), platelets and AFP post treatment but no significant change inHB Creatinine and TLC.

Table 3 Comparison between fibrosis scores before and after treatment.

\begin{tabular}{|l|l|l|c|}
\hline $\begin{array}{l}\text { Fibrosis } \\
\text { score }\end{array}$ & $\begin{array}{l}\text { Before } \\
\text { treatment }\end{array}$ & After treatment & p \\
\hline FIB4 & $2.053 \pm .774$ & $1.355 \pm 0.702$ & $<0.001$ \\
\hline APRI & $0.782 \pm 0.441$ & $0.418 \pm 0.339$ & $<0.001$ \\
\hline $\begin{array}{l}\text { Liver } \\
\text { stiffness }\end{array}$ & $8.866 \pm 1.744$ & $6.862 \pm 2.112$ & $<0.001$ \\
\hline
\end{tabular}

Table 3 showed that FIB4 score, APRI score and LS values were significantly lower after treatment in comparison the values before treatment. 
Table 4 Correlations between Liver stiffness [LS] and other study parameters.

\begin{tabular}{|l|l|l|}
\hline & \multicolumn{2}{|c|}{ LS } \\
\hline & \multicolumn{1}{|c|}{ R } & p \\
\hline FIB4 & $0.834^{* *}$ & 0.000 \\
\hline APRI & $0.799^{* *}$ & 0.000 \\
\hline BMI & $0.326^{*}$ & 0.029 \\
\hline Hb & $-0.322^{*}$ & 0.031 \\
\hline PLT & $-0.780^{* *}$ & 0.000 \\
\hline TLC & $-0.309^{*}$ & 0.039 \\
\hline ALT & $0.865^{* *}$ & 0.000 \\
\hline AST & $0.831^{* *}$ & 0.000 \\
\hline Albumin & $-0.775^{* *}$ & 0.000 \\
\hline Bilirubin & $0.810^{* *}$ & 0.000 \\
\hline Creatinine & $0.432^{* *}$ & 0.003 \\
\hline INR & $0.675^{* *}$ & 0.000 \\
\hline AFP & $0.779^{* *}$ & 0.000 \\
\hline
\end{tabular}

Table 4 shows that there were highly significant strong positive correlations between LS scores and FIB4, APRI score, ALT, AST, bilirubin, INR and AFP levels. Hb, Plts, TLC, and albumin were significantly negatively correlated to LS scores.

Table 5 Comparison between patients with and without LS improvement a regard age and BMI.

\begin{tabular}{|l|l|l|l|}
\hline & Improvement(no=30) & \multicolumn{1}{|c|}{$\begin{array}{c}\text { No improvement } \\
\text { (no=15) }\end{array}$} & p \\
\hline Age & $54.366 \pm 3.890$ & $55.20 \pm 4.616$ & 0.528 \\
\hline BMI & $23.366 \pm 3.727$ & $22.40 \pm 3.112$ & 0.393 \\
\hline
\end{tabular}

Table 5 shows that there were no significant differences between patients with and without improvement of LS after treatment as regard age and BMI.

Table 6 Rate of response among the study group.

\begin{tabular}{|l|l|l|}
\hline & No. & Percent \\
\hline Non-responder & 1 & 2.2 \\
\hline Responder & 43 & 95.6 \\
\hline Relapsing & 1 & 2.2 \\
\hline
\end{tabular}

Table 6 shows that $95.6 \%$ of the patients achieved complete response while one patient $(2.2 \%)$ was non-responder and one patients $(2.2 \%)$ was relapsing 
Table7 Comparison between fibro scan stages before and after treatment.

\begin{tabular}{|c|c|c|c|c|}
\hline \multicolumn{2}{|c|}{} & \multicolumn{2}{|c|}{ Fibro scan timing } & \multirow{2}{*}{ p } \\
\cline { 3 - 4 } \multicolumn{2}{|c|}{} & before treatment & $\begin{array}{l}\text { after 6 month of } \\
\text { treatment }\end{array}$ & \\
\hline & 0.00 & $0(0.0 \%)$ & $16(35.6 \%)$ & \multirow{3}{*}{$<0.00$} \\
\cline { 2 - 4 } & 1.00 & $5(11.1 \%)$ & $16(35.6 \%)$ & \\
\cline { 2 - 4 } Fibro scan stage & 2.00 & $29(64.4 \%)$ & $8(17.8 \%)$ & $5(11.1 \%)$ \\
\cline { 2 - 4 } & 3.00 & $10(22.2 \%)$ & $0(0.0 \%)$ & \\
\cline { 2 - 4 } & 4.00 & $1(2.2 \%)$ & & \\
\end{tabular}

Table 7 shows that the frequency of stages 0 and 1 was significantly higher after treatment while the frequency of stage 3 was significantly lower after treatment.

Figure 1 shows significant improvement of liver enzymes, liver synthetic functions (Albumin, bilirubin, INR), platelets and AFP post treatment but no significant change inHB Creatinine and TLC.
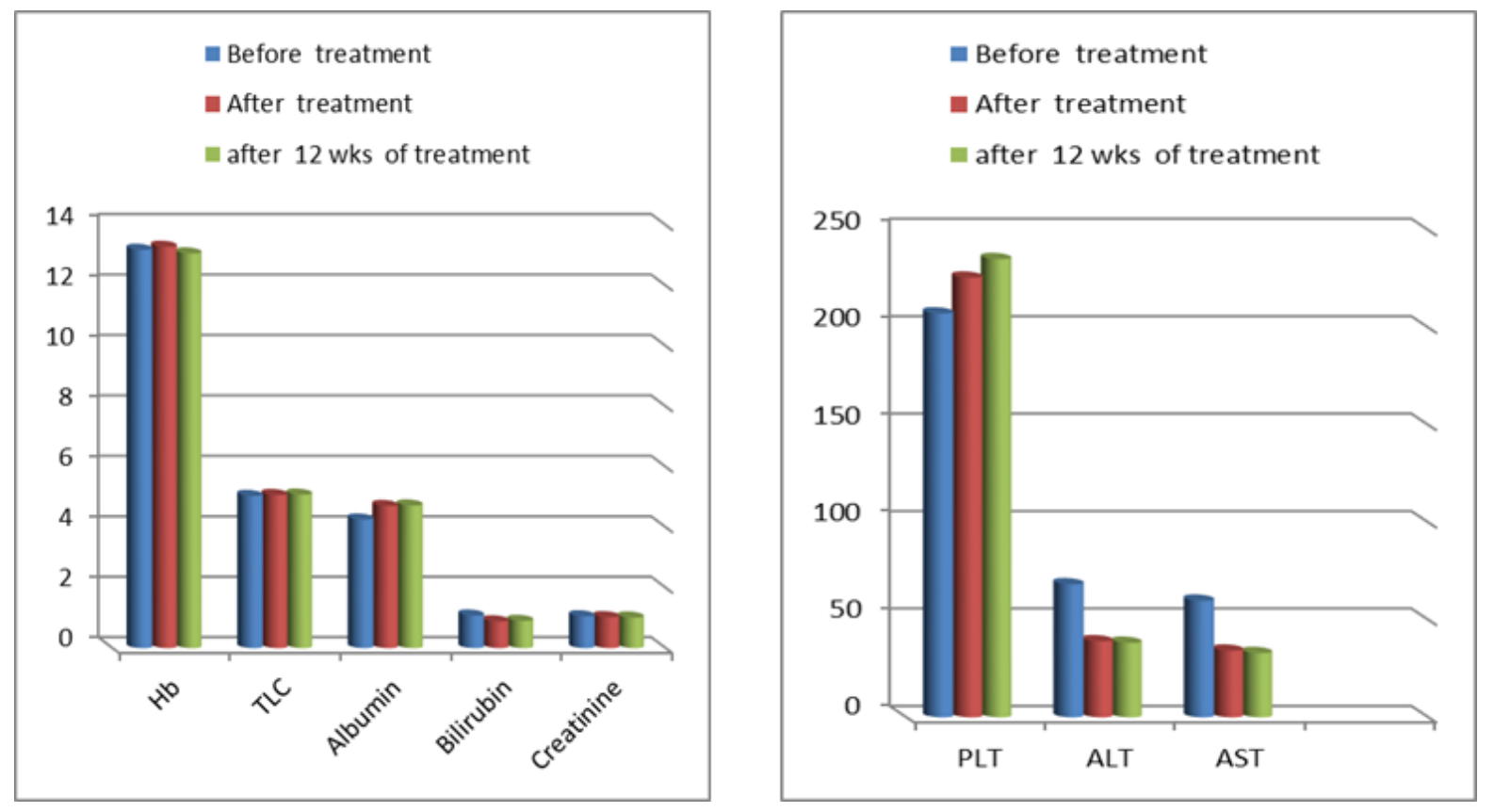

Figure 1 Comparison between laboratory data before and after treatment

Figure 2 Shows that the three score values were significantly lower after treatment in comparison the values before treatment. 


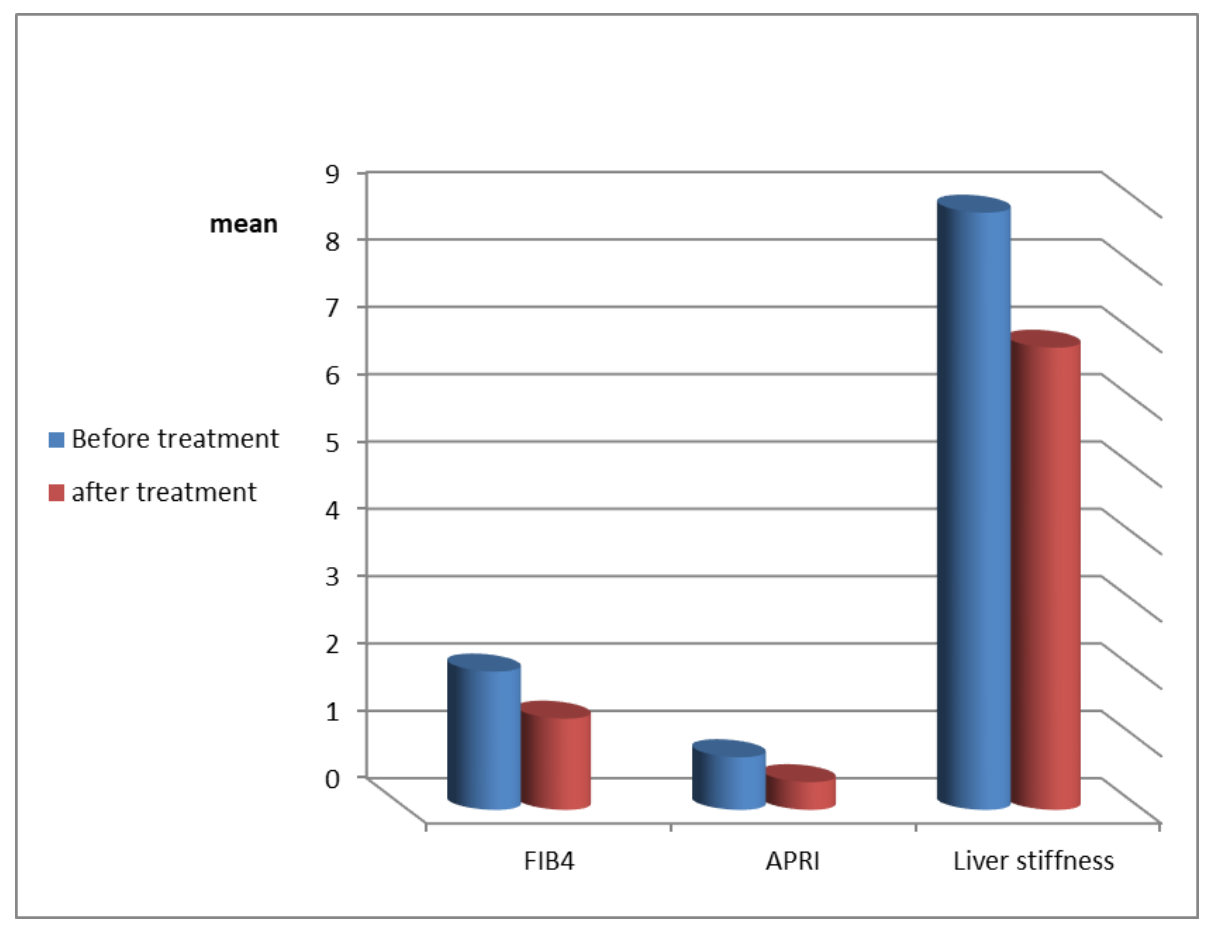

Figure 2 Comparison between fibrosis scores before and after treatment

Figure 3 shows that there were no significant differences between patients with significant fibrosis(f2-f4) and patients with nonsignificant fibrosis (f0-f1) as regard age and BMI.

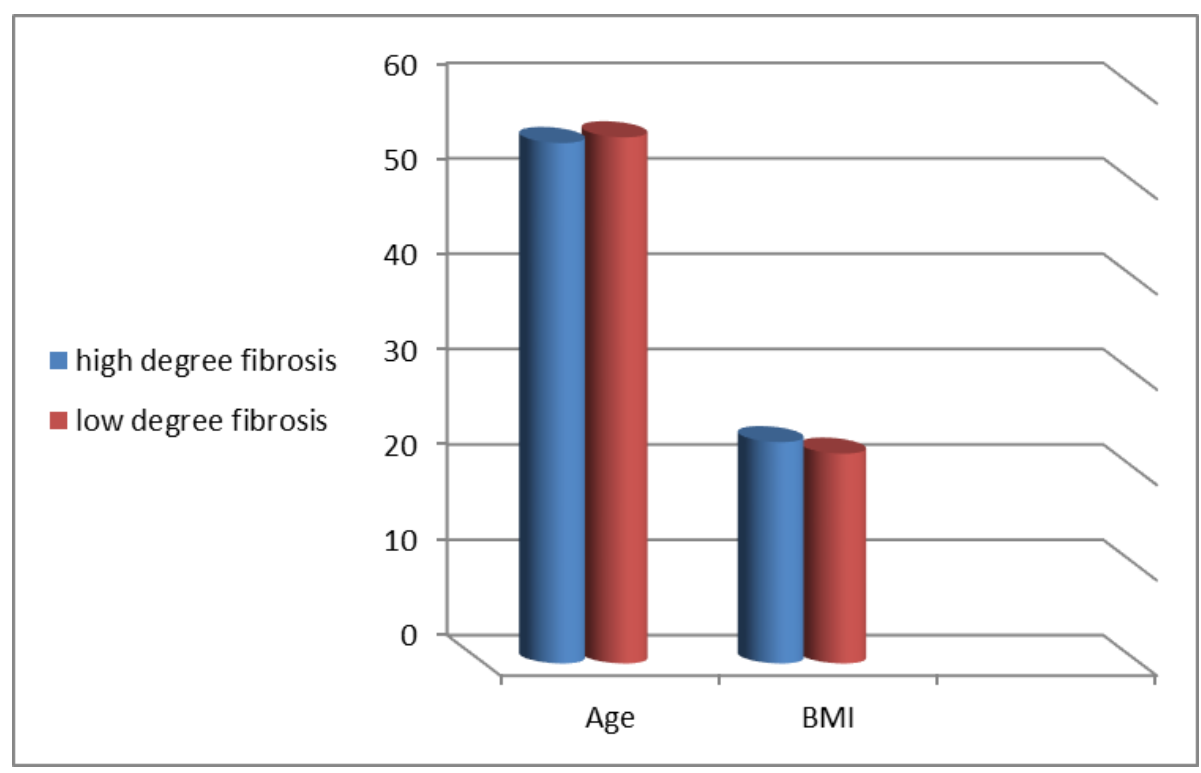

Figure 3 Comparison between patients with significant and nonsignificant fibrosis as regard age and BMI.

\section{Discussion}

The essential factor in the management of HCV disease is the impact on liver fibrosis and so the markedly improved SVR with DAAs and its impact on liver inflammation and fibrosis should be investigated.

As the major burden of chronic hepatitis $\mathrm{C}$ infection is progression of fibrosis and development of cirrhosis so the aim of this study is to evaluate the impact of Sofosbuvir-based regimens without INF on the changes of LS measurements by TE. 
Similarly, findings in this study are in agreement with different researches about fibrosis regression after DAAs. Similar to Martini et al. Study (5), the achievement of SVR was associated with improvement in fibrosis scores including APRI score, FIB4 index and LS by elastography.

The SVR achieved was $95.5 \%$ and this is similar to results of Brown et al study (6) which showed that SVR was 95\% when only patients who completed follow up were analyzed.

In this study Male sex was predominating among the study population (71.1\%) Elnadry et al. Study (7) showed that the sex distribution (male to female ratio in each group) in this study showed no statistically significant difference between the studied groups.

Similar to ElsharKawy et al., (2) this study reported a significant decline 12 weeks after EOT for LS measurements and fibrosis scores as FIB-4 and APRI. Also Sharma et al. approved that in their study which showed that liver stiffness was significantly decreased in patients with chronic HCV infection after successful treatment with 12 weeks of DAAs and those who achieve SVR, In addition also showed that there were a significant decrease in APRI score after 12 weeks of DAAs therapy which also correlates with the decrease in liver stiffness of these patients.

Similar to Omar et. al study(8), the results demonstrated significant serial improvements of FIB-4 index, APRI score and LSM by fibroscan along the follow up period $6 \mathrm{~m}$ after treatment in chronic HCV patients after DAAs treatment. Interestingly, these noninvasive fibrosis markers improved significantly regardless of the patients baseline fibrosis grade, fibrosis decreased in $66.6 \%$ of patients assessed using fibroscan 502 . Moreover the significant decline of the hepatic necroinflammation was evident by significant improvement of baseline necroinflammatory markers along the follow up period regardless of the patient baseline fibrosis grade. Also similar to this study, there were liver enzymes and platelets improvement throughout the follow up period and this improvement mirrored the changes and consequent improvement in fibrosis markers (FIB4 and APRI score).

This study was similar to yaras et al study(9) that showed that the decrease in mean LSM ( Kpa) was consistent with the improvement in viral and biochemical responses along with APRI and FIB4 score, this normalization of APRI and FIB4scores is mostly correlated with ALT and AST which are determinants of these scores.

Similar to Fan Hsu et. al , (10)these changes in APRI and FIB4 values might mainly result from the rapid decline in AST and ALT levels due to improvement in necroinflammation.

There were significant improvement in INR in patients with high degree fibrosis at end of treatment, this is similar to Pietsch et al study(11) which showed that TE improvements paralleled an increase in serum albumin, decrease in bilirubin andINR, indicating a continued improvement in liver function.

There were no significant difference in WBCs before and after treatment, However, Hablassi et al. Study (12) showed that the changes in liver fibrosis measurement were reflected as clinical improvement in laboratory parameters including WBCs.

There was no significant difference noted regarding pre and post treatment hemoglobin level which had mean values of 13.182 and $13.282 \mathrm{gm} / \mathrm{dl}$ before and after treatment respectively $(\mathrm{P}=0.44)$, this is similar to Menesy et.al study (13) that showed that there were no significant difference noted regarding pre- and post- treatment hemoglobin levels which had mean values of 11.4 and $10.84 \mathrm{gm} / \mathrm{dl}$ before and after treatment respectively. Conversely, El Sagheer et al. (14) reported a significant decrease in hemoglobin level from 13.6 before treatment down to $12.7 \mathrm{~g} / \mathrm{dl}$ after, Another study reported a significant change in hemoglobin level after DAAs treatment $(\mathrm{P}<0.001)$. It had mean values of 13.19 and $12.57 \mathrm{gm} / \mathrm{dl}$ before and after treatment respectively. Additionally, Alhaddad(15) and his coworkers also reported a significant change in hemoglobin level after DAAs therapy. It decreased from $12.28 \mathrm{gm} / \mathrm{dl}$ down to $10.09 \mathrm{gm} / \mathrm{dl}$ after treatment.

Mansour et. al, study (16) that showed that all patients in their studies whether cirrhotic or not cirrhotic showed a statistically significant decline in ALT, AST. Liver stiffness measurement. FIB4 score.,APRI score with significant improvement in platelet count in cirrhotic patients from baseline to SVR12 which goes with this study.

There was no differences between patients with high degree fibrosis and patients with low degree fibrosis as regard age and BMI, this results is similar to Guedes T et. al study(17) which showed that there was no significant differences regarding the demographic characteristics (Age and BMI), However, Soliman et.al study(18) said that there were effect 
of increased BMI on fibrosis regression in HCV patients and fibrosis scores regress more in patients with lower BMI. Also Fabbri et. al study(19) showed the changes in liver stiffness was significantly associated with the age of patient.

All patients who experienced decline in LSM during follow up experienced decline in ALT 12 weeks after EOT However, Brankenhoff et al. Study (20) which showed that there were no significant correlation between decline in LSM during follow up and decline in ALT 12 - 24 weeks after EOT.

Ebeid et.al, study (21) showed on comparing serum albumin level before and after treatment, there was decrease in albumin level after treatment.

Similar to Ali et al. Study, (22) laboratory data showed significant improvement in serum albumin and total bilirubin after treatment.

There was significant decrease in AFP level after treatment and there was highly significant strong positive correlations between changes in AFP and liver fibrosis parameters similar to Fouad et. al,(23) However, Jimenez et.al study showed that the severity of liver disease assessed by FIB4 or APRI scores was not associated with reduction in AFP over time.

There were significant reduction of fibrosis stages occurred in 22/29 ( $75.8 \%$ ) patients with F2 stage while reduction of fibrosis stage occurred in 5/11 (45.4\%) patients with F3 stage so percentage of enhancement in reduction of fibrosis stage in patient with F2 stage was more than percentage of enhancement in reduction of fibrosis in patients with F3 stage andthis goes with Abd Allah et al. (24)who documented significant reduction of fibrosis stages occurred in 14/16 ( $87.50 \%$ ) patients with F2 stage.

\section{Conclusion}

SVR achieved by DAAs therapy was $96 \%$ and was associated with significant improvement $(\mathrm{p}<0.05)$ of non-invasive fibrosis markers (FIB-4, APRI score, and LSM by Fibro Scan) from baseline compared to 6 months post-treatment. Using fibro scan regression of fibrosis in $30 / 45$ patients (66\%).

Regression of Liver inflammation and fibrosis in chronic hepatitis C patients post treatment with SOF DCV

\section{Compliance with ethical standards}

\section{Acknowledgments}

Thanks for all those who have helped in carrying out the research.

\section{Disclosure of conflict of interest}

Authors declare no conflict of interest.

\section{Statement of informed consent}

Informed consent was obtained from all participants.

\section{Statement of ethical approval}

Ethical approval was attained from the Committee of Research, Publications and Ethics of the Faculty of Medicine, BeniSuef University.

\section{References}

[1] ShoushaH, Mehassab M, Mehrez M, Afifi S, Elsharkawy M, Hamed M et.al. Noninvasive fibrosis scores and liver stiffness changes in chronic Hepatitis C after Sofosbuvir - based treatment. International Journal of Hepatology and Gastroenterology SCIRES literature. 2017; 3(4): 086.

[2] Elsharkawy A, Abdel Alem S, Fouad R, El Raziky M, El Akel W, Abdo M. Changes in liver stiffness measurements and fibrosis scores following sofosbuvir based treatment regimens without interferon. Journal of Gastroenterology and Hepatology. 2017; 32:1624 - 1630. 
[3] Mohammed Amin Mohammed 1, NesreenMoustafaOmarAssessment of Liver Fibrosis after Direct-Acting Antiviral Therapy in Compensated and Decompensated HCV-related Liver Diseases International Journal of Innovative Research in Medical Science (IJIRMS). April 2019; 04(04).

[4] El-Akel W, El-Sayed MH, El Kassas M, El-Serafy M, Khairy M, Elsaeed K et al. National treatment programme of hepatitis C in Egypt: hepatitis C virus model of care. J Viral Hepat 2017; 00:1-6.

[5] Martini S, Sacco M, Strona S, Arese D, Tandoi F, Dellolio D, et al. Impact of viral eradication with sofosbuvir based therapy on the outcome of post-transplant hepatitis c with severe fibrosis. Liver Int. 2017; $37: 62$ - 70.

[6] Brown S, Cheli N, Maheshwari A, Yoo H, Cook C, Zhang T et al. HCV compliance and treatment success rates are higher with DAAs in structured HCV clinics compared to general hepatology clinics. Medicine(Baltimore). 2019; 98(28):16242.

[7] Elnadry M, Abdel-AZiZ SA, Ghareb M, Ahmad AA, Abu Mohammed NM, et al. Impact of direct - acting antiviral therapy in Egyptian patients with chronic hepatitis c and liver cirrhosis. The scientific journal of AL - Azhar Medical faculty, Girls. 2017; 2: 181 - 188.

[8] Mohammed MA, Omar NM. Assessment of liver fibrosis after Direct Acting Antiviral Therapy in compensated and Decompensated HCV - related liver Diseases. International Journal of innovative Research in Medical Science (IJIRMS). 2019; 4(4): $256-263$.

[9] Yaras S, Sezgin 0, AlTintas E. Significant decrease in liver stiffness detected by two dimensional shear - wave elastography after treatment with direct - acting antiviral agents in patients with chronic Hepatitis C. Turk Gastroenterol. 2020; 31(2): 142 - 147.

[10] Fan Hsu W, Chou Lai H, Pang Su W, Hsin Lin C, Heng Chuang P, Hung Chen S, et al. Rapid decline of noninvasive fibrosis index values in patients with hepatitis c receiving treatment with direct acting antiviral agents. BMC Gastroenterol.2019; 19:63.

[11] Pietsch V, Deterding K, Attia D, Ringe KI, Gebel M, Manns MP.Long - term changes in liver elasticity in hepatitis C virus - infected patients with sustained virologic response after treatment with direct - acting antivirals. United European Gastroenterology journal. 2018; 6(8): 1188 - 1198.

[12] Hablassi FH, Lashen SA, Mohamed EA. Liver fibrosis Regression after Direct - Acting Antivirals for Hepatitis c virus: A prospective study. Journal of GASTROENTEROLOGY andHEPATOLOGy Research. 2021; 10(1): 3429 3434

[13] Menesy A, Ehab A, Abbas N. Impact of Direct - Acting Antiviral Agents Treatment on Body Mass Index and Lid Profile in Egyptian chronic Hepatitis c patients. Medical journal of viral Hepatitis (MJVH). 2021; 5(2): 21 - 26.

[14] El Sagheer G, Soliman E, Ahmad A, et al. Study of changes in lipid profile and insulin resistance in Egyptian patients with chronic hepatitis c genotype 4 in the Era of DAAs. Libyan Journal of Medicine.2018; 13(1).

[15] Alhaddad 0, wahb A, Sabry A et al. Role of Ribavirin in the Era of Direct - Acting Antiviral Therapies of chronic Hepatitis c. Expert review of anti - infective therapy. 2020; 18(8): 817 - 822.

[16] Mansour RH, Zaky S, El Kassas M, Mamdouh H, Esmat G. Evaluating the effect of direct - acting agents on liver fibrosis, by real - time elastography, Fibroscan and FIB4 score in chronic HCV patients. Al - Azhar Med Fac Girls. 2019; 3(1): 237-245.

[17] Guedes TP, Fragoso P, Lemos C, Garrido M, Silva J, et al. Long - Term follow up of Advanced Liver Disease after Sustained to Treatment of Hepatitis C with Direct - Acting Antivirals: Outcomes from a Real World Portuguese Cohort. GE Port J Gastroenterol. 2020; 27: 149 - 159.

[18] Soliman H, Ziada D, Salma M, Hamisa M, Badawi R, et al. Predictors for fibrosis Regression in Chronic HCV patients after the Treatment with DAAs: Results of a Real - World Cohort study. Endocrine, Metabolic \& Immune Disorders - Drug Targets. 2020; 20: 104 - 111.

[19] G Fabbri, I Mastrorosa, A Vergori, L Timelli, P Lorenzini, M Zaccarelli et al. Liver stiffness reduction and serum fibrosis score improvement in HIV / hepatitis c virus - coinfected patients treated with direct - acting antivirals. HIV Medicine. 2018; 19(8): 578 - 584.

[20] Brankenhoff SM, Verburgh ML, Willemse SB, Baak LC, Brinkman K, et al. Liver stiffness improvement in hepatitis c patients after successful treatment. The Netherlands Journal of Medicine. 2020; 78(6): 368 - 375. 
[21] Ebeid BA, Muhammed AA, Abd Elkareem SA, Soliman AS. Predictive value \& changes in child - Pugh score in chronic hepatitis c cirrhotic patients treated with Direct Acting Antiviral agents. Egyptian Journal of Medical Research. 2020; 1(2): 61 - 74.

[22] Ali OM, Hussein AA, Kholef EF, Elsweify WA.Effect of sofosbuvir plus daclatasvir on virological response and liver function tests as a line of treatment for HCV related cirrhosis. A prospective cohort study. Egyptian liver Journal. 2020; 10: 27.

[23] Fouad R, ElsharKawy A, Abdel Alem S, El Kassas M, Sweedy A, Afify S et al. Clinical impact of serum serum alfa fetoprotein and it's relation on changes in liver fibrosis in hepatitis c virus patients receiving direct acting antivirals. Eur J Gastroenterol Hepatol. 2019; 31(9): 1129 - 1134.

[24] AbdAllah O, Ghoneem E, Eissa M. Liver Fibrosis in Assessment in cases of Chronic Hepatitis C after Direct - Acting Antivirals Therapy using Aspartate Aminotransferase to platelet Ratio Index and Transient Elastography. Afro Egyptian Journal of Infectious and Endemic Diseases. 2021; 11(1): 10 - 17. 